\title{
Electric and Magnetic Properties of Graded Oriented Polymer Composites
}

\author{
Jimsher Aneli, Levan Nadareishvili and Lana Shamanauri \\ Institute of Machine Mechanics, 10 Mindeli Str., Tbilisi 0186, Georgia
}

\begin{abstract}
The character of variations of the local electric resistance and magnetic susceptibility of film polymer composites with graded anisotropic structures on the basis of polyvinyl alcohol, graphite and nickel nano-powders have been studied. Graded anisotropic structures are formed after orientation (stretching) in spatial conditions of thin polymer composites. Structure anisotropy leads to anisotropy both electrical conductivity and magnetic properties of these films. It is established that the changes of these parameters essentially depend on both initial shape of the films and on direction of their orientation. So, after orientation of the rectangle films parallel to it any side forms the film, electrical resistance of which in parallel and rectangle directions to orientation axis changes by Gauss low. The composites containing the magnetic particles after orientation are characterized with analogical regularity (extreme dependence of the magnetic susceptibility on the coordinates of the film along and perpendicular to stretching directions). Gradient distribution of the local resistances and magnetic susceptibilities in the stretched films along and rectangle to stretching direction is due to gradient of local deformations in the same directions. In electronics these films can be useful for preparing of multifunctional printed circuits of new generation.
\end{abstract}

Key words: Polymer films, composites, stretching, anisotropy, local electrical resistance, magnetic susceptibility.

\section{Introduction}

It is well known that there are several methods for obtaining of materials with anisotropic properties by chemical methods (copolymerization, polymer-analogous transformation, radiation-chemical modifications, etc.) [1, 2]. At present for obtaining of such structures, one of the best methods is the orientation of polymer films in the definite direction and environment conditions. It is known also that at stretching of thermoplastic polymers above glass temperature the material in orientation state is formed. Such polymers are characterized with mono-axis crystal symmetry. In this state the principal direction of macromolecules coincides with the direction of stretching. In the polymer filled with different dispersive fillers, particularly with electric conductive and magnetic materials (graphite, carbon black, metal

Corresponding author: Jimsher Aneli, professor, research fields: electric conducting polymer composites, different sensors on the basis of polymer materials, radiation-resistant polymer materials. powders), the particles distribution of lasts interacting with macromolecules transform from chaotic state to orientation one. The change of polymer microstructure significantly defines the material electric and magnetic properties $[3,4]$.

In the presented work the character of change of electric conductivity and magnetic susceptibility of polymer composite films based on polyvinyl alcohol, graphite and nickel at their mechanical stretching has been investigated.

\section{Results and Discussion}

The films were prepared with using of following technology:

The water solution of fine grained graphite (average diameter of grains less than 10 micrometers) or nickel (average diameter of grains less than 20 nanometers) suspensions in polyvinyl-alcohol was prepared. The mixture was filtrated through the filter with diameter about $10 \mathrm{~cm}$ and the film was formed on the dryer table. 
The specific volumetric electric resistance of the polymer films was changed in the interval 10-50 $\mathrm{kOhm} . \mathrm{cm}$. The selection of such interval of the composite resistance was dictated by preliminary selection of conducting composites effectively reacted on the mechanical deformations [4]. The experiments were carried out on the basis of polymer composite films with rectangle and trapezoidal shape. The thickness of films was no more than $0.2 \mathrm{~mm}$. The deviation of the values of resistance for any local region of the film was no more than $10 \%$. These films were fixed in special clamps, placed to the heater and were stretched on $50-150 \%$ at rate $50 \mathrm{~cm} / \mathrm{min}$ and temperatures $100-120{ }^{\circ} \mathrm{C}$. Stretching was conducted for rectangle form sample along big side and for trapezoidal sample in parallel to bases direction (Fig. 1).

After stretching of the deformed films, local electric resistances were measured. First of all, it was necessary to mark the film with square grid. In our case the length of square side was equal to $5 \mathrm{~mm}$. The local resistance of each element of the grid was measured by using of twin needles after touching them to the film (two wires measurement). The deviation of the values of resistance for any local region of the film was no more than $10 \%$.

Another series of investigations was conducted on establishment of local magnetic characteristics of graded-anisotropic magnetic polymeric composites with nickel powders. The films made of magnetic polymer composites were subjected to similar stretching procedure as it was previously described.

The resulting distribution of magnetic particles density in polymer composites was recorded by the method of LC-generator similar to the one used by us in Ref. [5] for NMR detection in europium garnet at low temperatures.

It is also interesting to note that a similar method was used for the first precision determination of the magnetic field penetration length in superconductors [6].
For assessment of magnetic susceptibility distribution over the surface of gradient magnetic films, an LC generator with sinusoidal oscillations was used [5].

The procedure of measurements of the magnetization changes is realized through the sensitive detection of the change of inductance of the LC generator oscillatory contour coil supplied with a tipped ferrite rod core at scanning by it over the investigated surface (Fig. 2). In whole, the susceptibility measurement system consists of the following stages: LC generator, emitter follower for cascade matching, sinusoidal oscillation amplifier and frequency meter.

The frequency of LC generator oscillatory contour is defined by its fixed total capacitance and inductance. The oscillatory contour is an isolated system and tuned on a reference frequency providing its operation on the maximal sensitivity as result of which the slightest contour inductance changes (induced by change of local

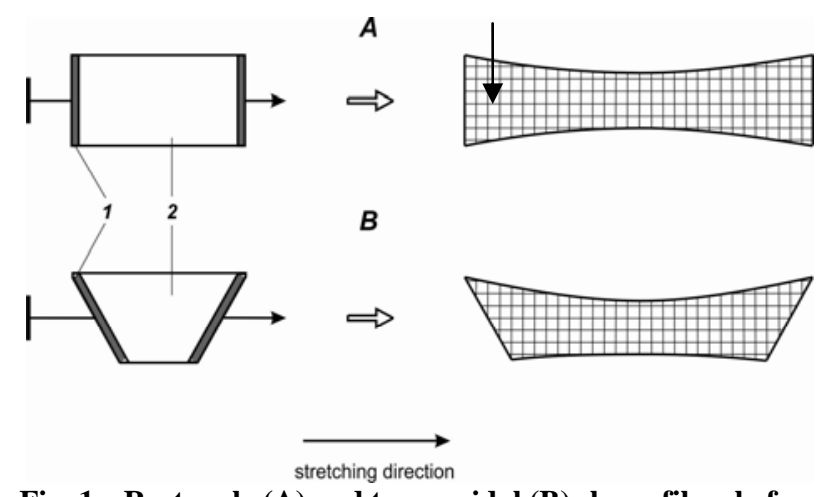

Fig. 1 Rectangle (A) and trapezoidal (B) shape films before (left) and after (right) stretching along big sides (with applied grids after stretching). 1-captures; 2-film (sample).

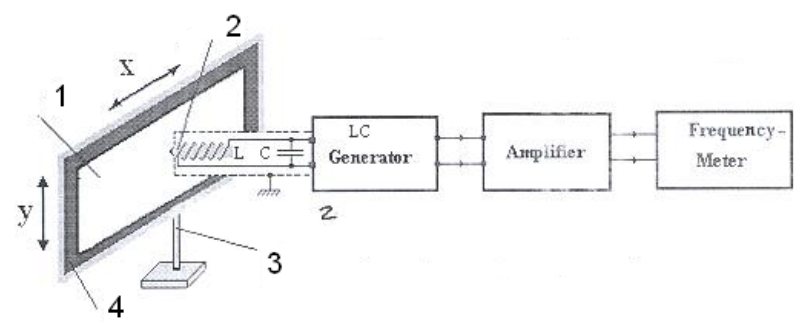

Fig. 2 Scheme of the measuring of the magnetic characteristics of the polymer films. 1-Sample; 2-ferrite tip; 3-support; 4-frame. 
concentration of magnetic particles in the film) lead to the significant changes of contour frequency.

Let us note that the induction coil of oscillatory contour was brought away on $30-40 \mathrm{~cm}$ from the LC generator by a screened transmission line and the contour inductance with its spurious capacitance enters into contour effective parameters. The frequency detuning is possible using a $2 \mathrm{~mm}$ thick and $15 \mathrm{~mm}$ long tipped ferrite core put into the coil during its scanning over the film. Namely this principle is used for the definition of the contour quality factor change expressed in the frequency units. During the displacement of coil having a tipped ferrite core near the surface of the gradiently magnetized film surface the change of coil inductance takes place which is followed by the corresponding change of LC generator frequency in a quite significant range giving one possibility to evaluate the change of value of the real part of susceptibility related to frequency.

The experimental set-up is presented in Fig. 2. In the inductive coil of the resonance contour of LC-generator, it is placed cylindrical tipped ferrite rod used as probe. The investigated rectangular shape magnetic polymer composite film is displaced relatively the immovable ferrite tip. The scanning of the film surface is realized along the previously marked net contour (Fig. 3).

Measurements of the dependences $F-1$ were provided with using of the ferromagnetic film, the scheme of which is presented in Fig. 3.

The change of magnetic particle concentration causes the change of inductance $\delta \mathrm{L}$ of the resonance contour of LC-generator resulting in the frequency displacement of LC-generator $\delta$ f related with $\delta \mathrm{L}$ by relation:

$$
\delta \mathrm{f} / \mathrm{f}=1 / 2 \delta \mathrm{L} / \mathrm{L} .
$$

This frequency displacement could be precisely measured what stipulates the high sensitivity of the method.

At the natural frequency of used LC-generator near $2 \mathrm{MHz}$ the observed range of the frequency change $\delta \mathrm{f}$ was about $\sim 1,000 \mathrm{~Hz}$ at the precision of the frequency measurement $\sim 1 \mathrm{~Hz}$.

As a result of measuring the local resistances of oriented along the parallel to long side of the rectangle shape samples it was established that maximum change of this parameter was noticed along symmetry axes of the rectangle along orientation direction. This change has an extreme character (the maximum is at the central part of the film) and its full shape has Gaussian form. Fig. 4 shows that the maximum heights of the local resistances depend on the value of stretching. This result is in good agreement with the known conception on the mechanism of conductivity of conductive polymer composites [3]. Investigation of obtained films using of the metallographic microscope shows that the average optical density of the penetrated light through the film nearly exponentially depends on the stretching direction. Therefore it may be proposed that the dependence of concentration of the conducting particles in the local regions of the film has the same character. However the basis of such distribution of the filler particles in the uniaxially stretched polymer composites is not yet clear.

By analogical shape of the same dependence is characterized the local resistances in the same film rectangle to stretching directions, although these dependences are somewhat weaker.

It was interesting to establish the character of considered above functional dependences of the local resistances for composites with different filler concentration. Fig. 5 shows that the increase of filler concentration leads to reduction of the intensity of

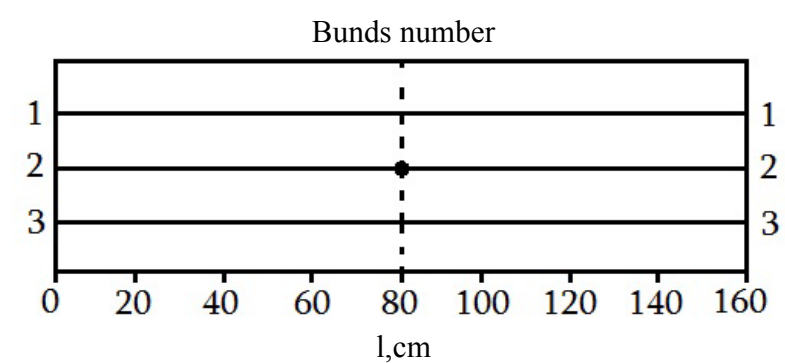

Fig. 3 Scheme of ferromagnetic film for investigation of the local magnetic susceptibility along stretching directions in bands 1-1, 2-2 and 3-3; dotted line- the middle of the film. 


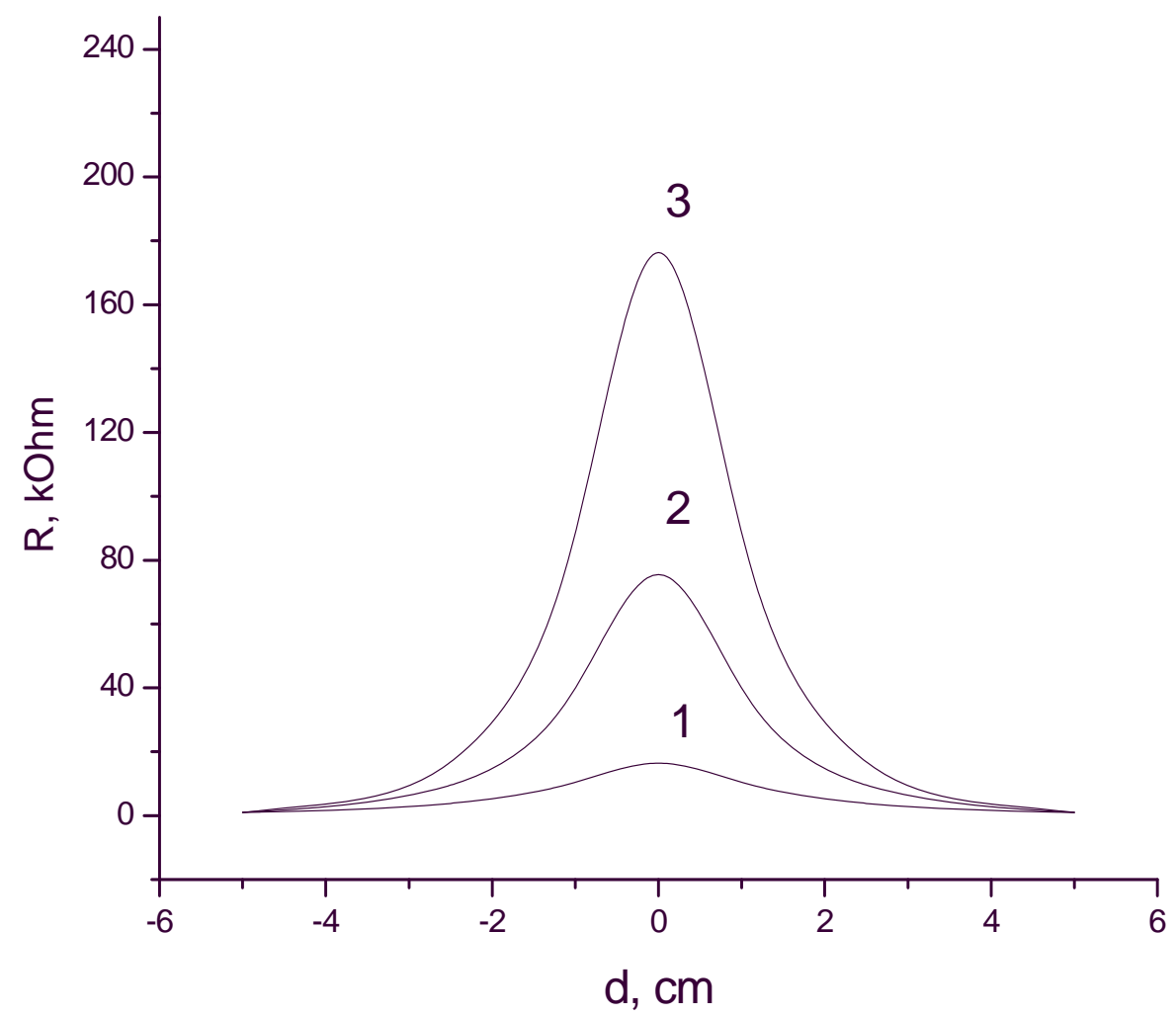

Fig. 4 Dependence of local resistances of polymer film on the value of stretching parallel to long side of the rectangle on 50 (1), 100 (2) and $150 \%$ (3). The maximums of curves are located on the central line with abscissa coordinate " 0 ".

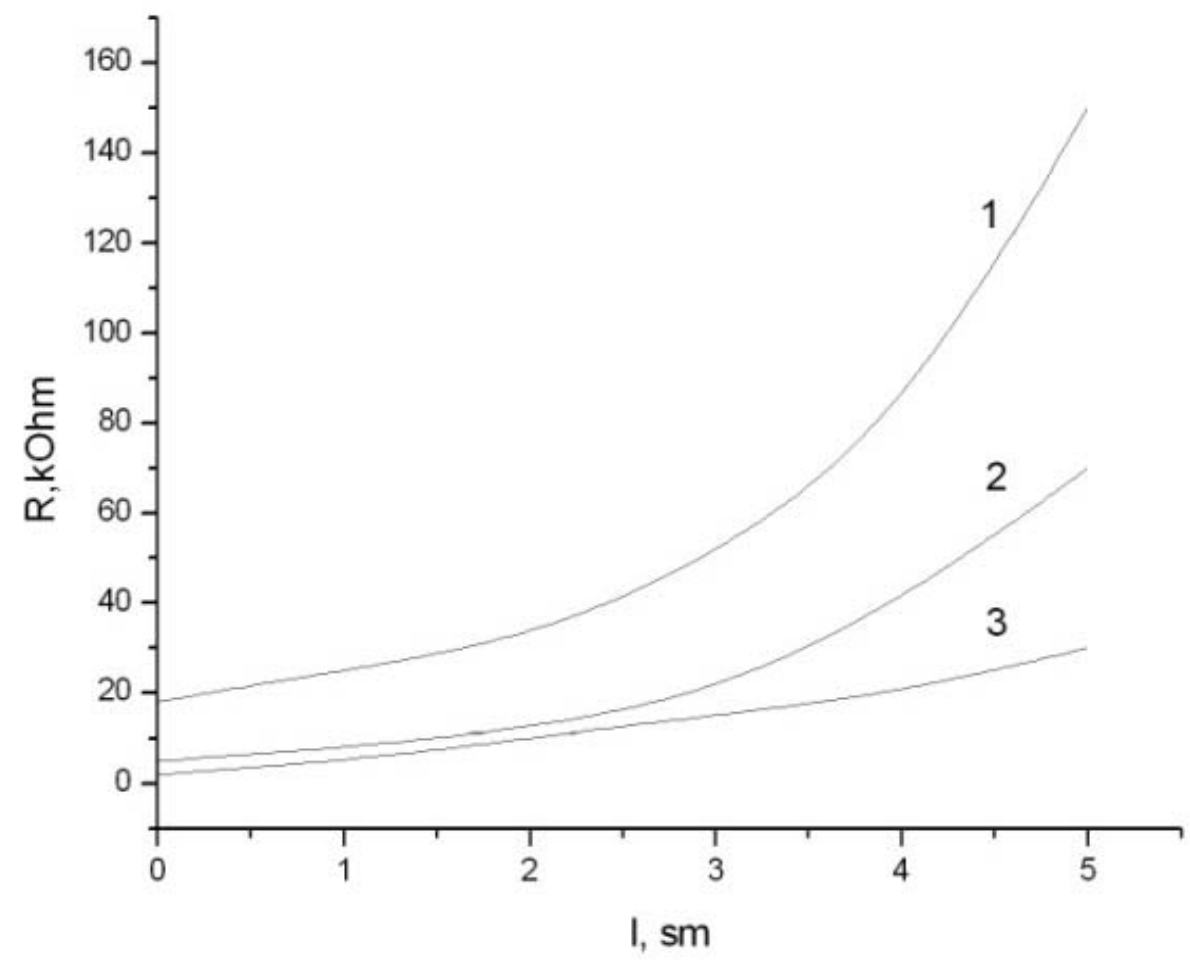

Fig. 5 Dependence of local resistances of films on the stretching degree for composites based on PVA containing 30 (1), 40 (2) and 50 (3) mas.\% of graphite powder (curves correspond to left half side of central strip of the film). 


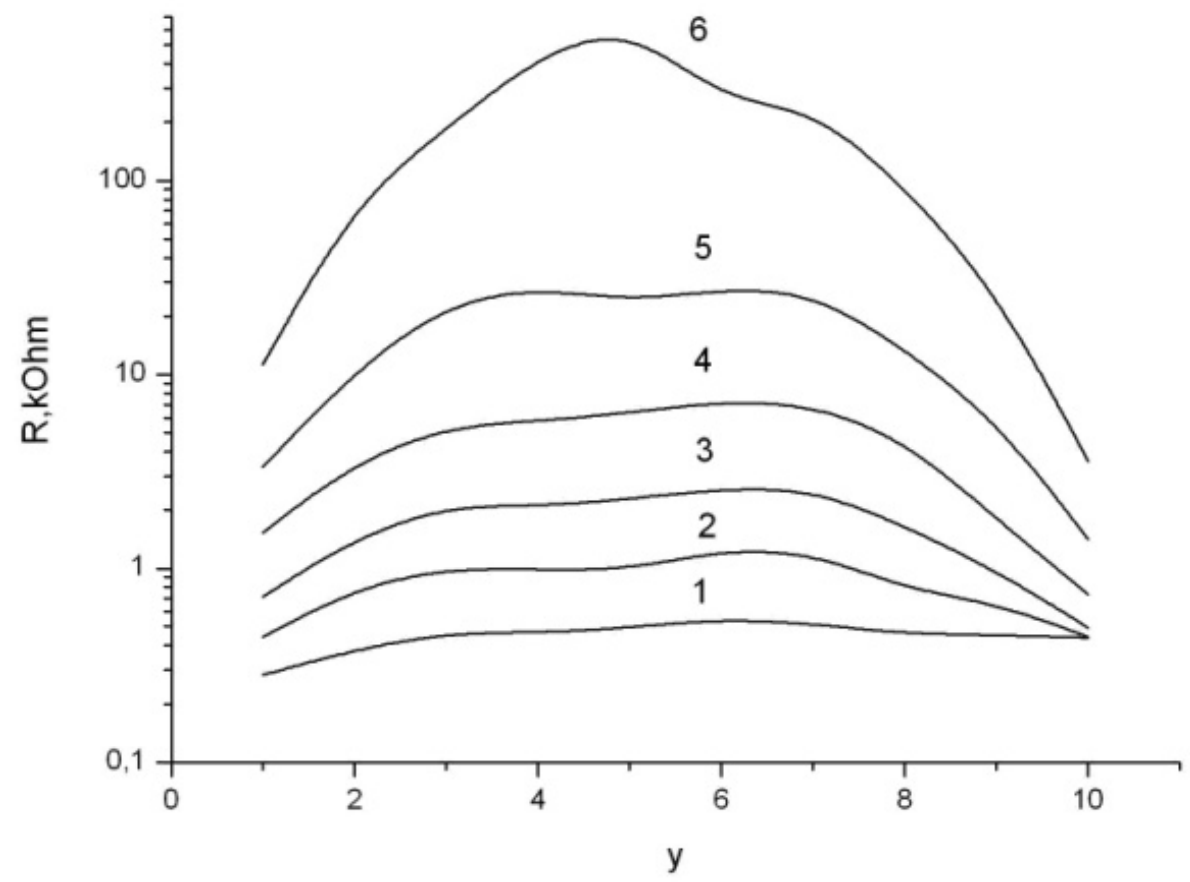

(a)

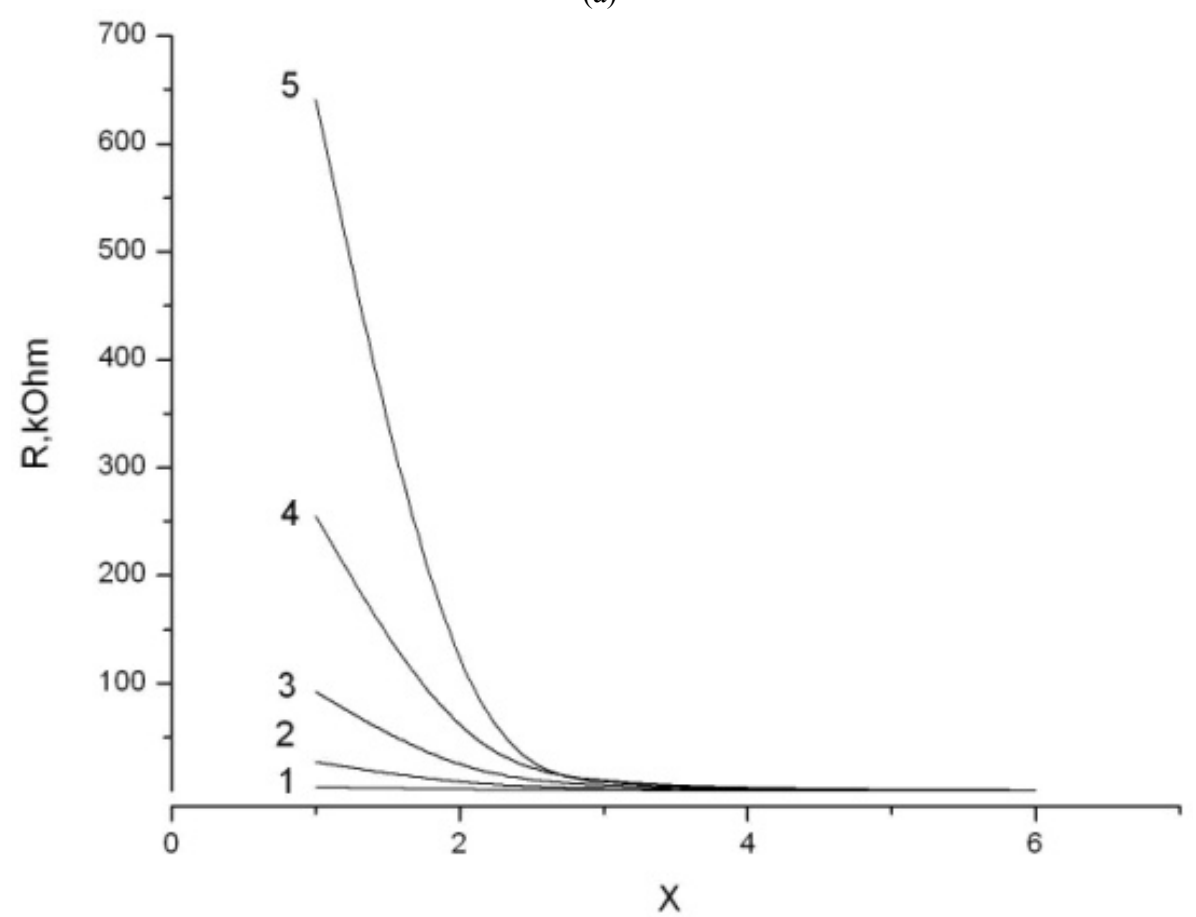

(b)

Fig. 6 Dependences of local resistances on the film (PVA with 25 mas.\% graphite) coordinates in the strips stretched on $150 \%$ parallel to bases of trapezoidal shape films in perpendicular to stretching direction from big base to small one (a) and parallel to stretching direction from small base to big one (b). The numbers on the curves indicate the numbers of stripes in perpendicular (a) and parallel (b) to stretching direction. The curve number 5 of Fig. 6, (b) corresponds to central strip of trapezoidal film and others-to side from it ones (4-3-2-1). The asymmetry of the curves on the Fig. 6, (a) is due to certain inhomogeneous distribution of the filler particles in polymer matrix.

resistance change at stretching. This phenomenon may be described by following processes. It is known that the filler particles at stretching of polymer composites commit the mutual transition in the polymer matrix 
initiated by interactions between them and macromolecules, as result of which the average distances between these particles and consequently the process of charge transition change accordingly [7]. Here the inverse process - approach and removing of the particles and consequently the probability of creation of the conducting chains changes respectively. However in case of composites with high concentrations of the conducting particles the probability of arising of new contacts between ones is higher than in opposite case, since the frequency of these elementary processes is higher than in case of composites with relatively low concentrations of these particles. Here the described process is analogical to reserving of the switching conducting chains in the complex electrical engineering schemes. The obtained results show that the process of displacement of the conducing particles and consequently the change of conductivity take place more intensively in the middle part of the stretched polymer film than in other ones, namely, this change has gradient character with increasing from grips till middle of the film. The higher is the amount of this gradient, the lower is the filler concentration.
The following series was fulfilled on the trapezium shape conducting films. Here was created the mechanical stretch gradient in perpendicular to base direction, along which the stretching was realized. This gradient was increased from big base and was ended at more stressed small base with maximum.

The experimental data on the definition of character of the dependence of distribution of the local resistances on stretched both rectangle and trapezoidal form films on their coordinates show that the shape of these dependences essentially is defined mainly by two factors: (a) the content of electrical conducting fillers in the selected part of the stretched film and (b) distribution of conducting particles in the polymer matrix after stretching along selected direction. Nonlinearity of the dependence of local electrical conductivity on coordinates both in along and perpendicular directions of stretching is explained mainly by the nature of the conductivity of the polymer composites filled with conducting particles. Namely it is well known that the charge transfer in such systems obey to tunnel mechanism-nonlinear dependence of the conductivity on the distance between neighbor particles $[3,4]$.

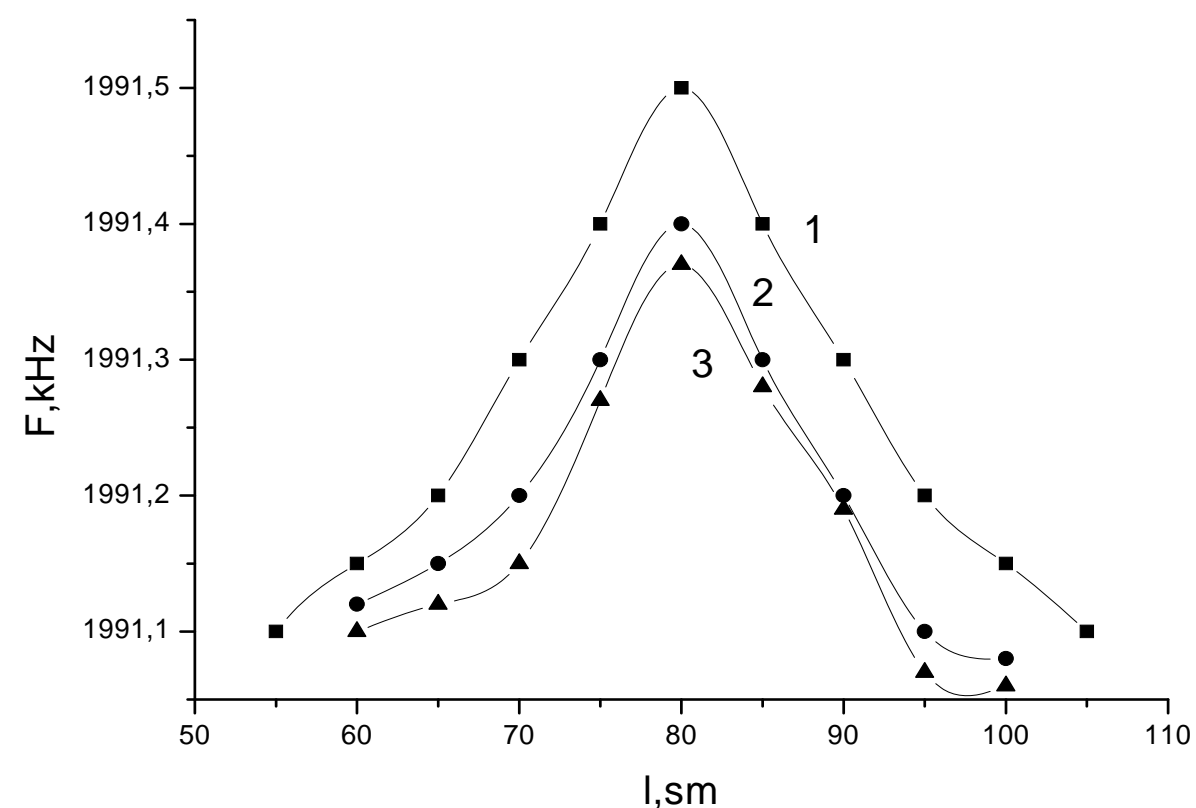

Fig. 7 Dependences of the oscillation frequency of the ferrite sensor on the coordinates of film made from polymer composite along directions 2-2 (1), 1-1 (2), 3-3 (3). 


\section{Graded Anisotropic Magnetic Polymeric Composites}

In Fig. 7, the results of measurements of generator frequency change along contour lines 1-1, 2-2 and 3-3 are presented. Similar results are obtained at measuring of magnetic susceptibility of film along rectangular to 1-1, 2-2 and 3-3 directions.

The presented results show that for magnetic polymer composite films with rectangle shape the dependences $\mathrm{F}-1$ have similar form, as was obtained for conducting polymer composites $(\mathrm{R}-1)$ with same shape. Such resemblance is based on similar character of distribution of the filler particles - conducting ones in rectangle electrical conducting polymer composites and magnetic particles in the magnetic analogues after their uniaxial stretching, because the maximums of curves of dependences of the local resistance on film coordinates correspond to rarefied regions of these films and the maximums of the curves $\mathrm{F}-1$ correspond to those regions - frequency increases, when magnetic particle concentration decreases and, consequently, local susceptibility and inductance decrease.

Results of detailed measurements will be published elsewhere.

\section{Conclusion}

Gradiently anisotropic structures are formed after orientation (stretching) in spatial conditions of thin polymer composites based on polyvinyl alcohol, graphite and nickel powders with electrical conducting and magnetic properties. Structure anisotropy leads to anisotropy both electrical conductivity and magnetic properties of these films.

After orientation of the rectangle films parallel to it any side forms the film, electrical resistance of which parallel and rectangle directions to orientation axis changes by Gauss low.

Gradient distribution of the local resistances in the stretched films along and rectangle to stretching direction is due to gradient of local deformations in the same directions.

The gradient distribution of magnetic particles in the stretched direction leads to equivalent change of magnetic properties of these films.

The experiments described above open the perspectives in the field of creation of the films with desirable anisotropy of electric conductance and magnetic properties. The perspective is in application of these materials for creation of the so called printed schemes. In electronics these films will be useful for preparing of multifunctional micro-schemes.

\section{References}

[1] Lekishvili, N. G., and Nadareishvili, L. I. 2002. "Polymers and Polymeric Materials for the Fiber and Gradient Optics/VSP.” Utrecht-Boston-Köln-Tokyo, 230 (Monograph).

[2] Nadareishvili, L. I. 2004. "GB-Optics-A New Direction of Gradient Optics.” J. Appl. Pol. Sci. 91: 489-93.

[3] Aneli, J. N., Khananashvil, L. M., and Zaikov, G. E. 1998. "Structuring and Conductivity of Polymer Composites." Nova Sci. Publ. N. Y. 326 (Monograph).

[4] Aneli, J. N., Khananashvil, L. M., and Zaikov, G. E. 1999. "Effects of Mechanical Deformations on Structurization and Electric Conductivity of Polymer Composites." J. Appl. Pol. Sci. 74: 601-21.

[5] Pavlov, G. D., Chekmarev, V. P., Mamniashvili, G. I., and Gavrilko, S. I. 1988. Method of NMR Recording in Magnetoordering Materials. USSR Patent No. 279893.

[6] Schawlow, A. L., and Devlin, G. E. 1959. "Determination of the Magnetic Field Penetration Length in Superconductors." Physical Review 113 (1): 120-6.

[7] Aneli, J. N., Zaikov, G. E., and Mukbaniani, O. V. 2011. "Physical Principles of the Conductivity of Electrical Conducting Polymer Composites (Review)" Chemistry and Chemical Technology 5: 75-82. 